\title{
Commentary
}

\section{Ethical Considerations for Acupuncture and Chinese Herbal Medicine Clinical Trials: A Cross-cultural Perspective*}

\section{Christopher Zaslawski}

College of Traditional Chinese Medicine, Department of Medical and Molecular Biosciences, Faculty of Science, University of Technology, Sydney, Australia

\begin{abstract}
Many ethical concerns revolve around the four basic principles of research: merit and integrity, respect for human beings, weighting of risk-benefit and justice. These principles form the basis for any discussion concerning human research ethics and are applicable to all areas of research including acupuncture and Chinese herbal medicine. World Health Organisation document, Guidelines for Clinical Research on Acupuncture, states that 'consideration should be given to the different value systems that are involved in human rights such as social, cultural and historical issues' and that 'further studies should be conducted in relation to ethical issues involved in clinical research on acupuncture'. In addition to outlining the four basic principles, this paper will also examine the effect of Asian culture on Western human research ethics and how this may impact upon issues such as informed consent and weighting of risk-benefit.
\end{abstract}

Keywords: acupuncture - Chinese medicine - culture - ethics - human research

\section{Introduction}

Over the past three decades there have been many developments concerning acupuncture and Chinese medicine $(\mathrm{CM})$ research methodology (1). In addition, there has been increasing interest, especially in Western countries, on the consideration of ethics on research design (2). The World Health Organisation document, Guidelines for Clinical Research on Acupuncture (3), states that 'consideration should be given to the different value systems that are involved in human rights such as social, cultural and historical issues' and that 'further studies

For reprints and all correspondence: Christopher Zaslawski, College of Traditional Chinese Medicine, Department of Medical and Molecular Biosciences, Faculty of Science, University of Technology, Sydney 2007, Australia. Tel: +61-2-9514-7856; Fax: +61-2-9514-7866;

E-mail: chris.zaslawski@uts.edu.au

*Earlier versions of this paper were presented at the Third World Congress of Chinese Medicine, Hong Kong November 2006 and at the workshop 'Authenticity, Best Practice and the Evidence Mosaic-The Challenge of Integrating Traditional East Asian medicines into Western Health Care' at the University of Westminster, London, April 2007. should be conducted in relation to ethical issues involved in clinical research on acupuncture'.

Most Western and an increasing number of Asian countries now require that research projects involving humans have independent ethical review prior to initiating research. A committee, often comprising medical and non-medical persons, usually conducts these reviews. The reviews are undertaken to ensure the protection of the welfare and rights of research participants as well as the facilitation of research that will benefit society and humankind. In the United States, these committees are called Independent Review Boards, while in the United Kingdom they are termed Research Ethics Committees and in Australia, Human Research Ethics Committees. Indeed, many funding bodies require that such a committee approve proposals before releasing funding for the research. In addition, many ethics committees require that research conducted overseas under the direction of local institution comply with the local ethical requirements. The Declaration of Helsinki, an international set of guidelines for ethical research adopted by the World Medical Association, is clear in enunciating the need for a committee to review research proposals involving humans (4). 


\section{Paragraph 13 states}

This protocol should be submitted for consideration, comment, guidance and where appropriate approval to a specially appointed ethical review committee, which must be independent of the investigator, the sponsor or any kind of undue influence.

\section{Paragraph 9 reiterates}

Research Investigators should be aware of the ethical, legal and regulatory requirements for research on human subjects in their own country as well as applicable international requirements.

Many ethical concerns revolve around the four basic principles of research merit and integrity, respect for human beings, weighting of risk-benefit and justice. These principles form the basis for any discussion concerning human research ethics and are applicable to all approaches (quantitative or qualitative) and types (clinical or non-clinical) of research including CM.

\section{Research Merit and Integrity}

Establishing research merit and integrity is necessary for the ethical involvement of human participants. The trial design should use recognized principles of research with tangible, clinically relevant measurable outcomes. The unique characteristics of the clinic practice of $\mathrm{CM}$ such as pattern differentiation and individualization of treatment make it difficult to translate into accepted research models. This can pose difficulties for the ethics committee if they are unfamiliar with the principles of CM, as well as for the researchers who may submit proposals with novel design innovations to accommodate such clinical practice characteristics (5).

Defining the research question and engaging in ongoing dialogue with the ethics committee are important strategies if there is to be a meaningful and successful relationship. Both the researchers and the ethics committee will have to learn from one another if they are to fulfill their obligations (6). This can be a new experience for $\mathrm{CM}$ practitioners who may be undertaking research for the first time, are unaccustomed to operating within a Western research paradigm and who may find the process of seeking ethics approval intimidating.

An important issue relating to integrity involves the existence of equipoise. Equipoise has been defined as 'a state of genuine uncertainty on the part of the clinical investigator regarding the comparative therapeutic merits of each arm in a trial' (7). Small preliminary pilot studies or effectiveness studies can be very informative in establishing equipoise. Fønnebø and colleagues (8) have recently suggested a phased research activity sequence that included safety status and randomized controlled pragmatic studies prior to commencing efficacy-focused research. Preliminary pilot studies have also been undertaken in the context of efficacy studies. A recent example is the series of knee osteoarthritis studies conducted by Berman and colleagues (9-11). Failure to have conducted a preliminary study that showed possible benefit (equipoise) may not only waste public grant monies but also the participant's time and efforts. Research therefore should be staged to justify the efficient use of public monies and participant's contribution.

In order to be ethical, research must be conducted or supervised by persons or teams with the experience, qualifications and competence appropriate to the research. An important consideration for 'hands on' type research such as acupuncture is the skill and competence of the acupuncturist administering the interventions. Unfortunately, some studies fail to use qualified, competent practitioners. Furthermore, the acupuncturist should have experience in the treatment of the condition being studied. When reporting the study, the qualification, the length of training and clinical experience of the acupuncturist should be stated.

In addition, the results of research and the methods used should be published in ways that permit scrutiny. This means full reporting of all stages of the research. A number of international standards exist that gives guidance and provide checklists for improving study design. A good example is the Consolidated Standards of Reporting Trials (CONSORT) statement that lists 22 items for improving the quality of parallel group randomized trials (12). More recently, guidelines have been developed for reporting acupuncture and Chinese herbal medicine research (13-15).

Dissemination of research results is another often overlooked ethical issue. Clinical research that has a negative outcome is frequently not submitted for publication. In addition, journal editors may have a bias against publication of a negative outcome trial. Reporting of negative trials should be encouraged. Failure to do so has been blamed for distorting the medical literature. The Declaration of Helsinki (paragraph 27) (4) is explicit in this regard, stating that

Both authors and publishers have ethical obligations. In publication of the results of research, the investigators are obliged to preserve the accuracy of the results. Negative as well as positive results should be published or otherwise publicly available.

The open access BMC (Biomed Central) Journal of Negative Results in BioMedicine represents a very novel approach to dealing with editorial bias (16).

Finally, the acknowledgement of sponsorship and conflicts of interest should be reported as conflicts involving personal, professional or financial situations can actually or potentially affect judgments. Of all 
Medline listed complementary and alternative medicine journals, only two require such a declaration (17).

\section{Respect for Human Beings}

The second issue of respect for human beings requires that participants choose whether or not to become involved in a research study. Central to this concept is the participant's informed consent.

General requirements for consent involve:

- Voluntary participation that is based on sufficient information and understanding of the purpose, methods, demands, risks, inconvenience, discomforts and possible outcomes of the research

- That the participant is not subject to coercion or to inducement and that the participation is clearly established such as a signed form, return of a survey or a recorded audio agreement.

In many Western countries, informed consent is obtained by using a document that is signed by both the participant and the researcher. However, a recent review of acupuncture trials found that of the 47 shamcontrolled acupuncture trials reported, only 10 studies included information on how participants were informed (18). They also found that information strategies varied considerably and were often not fully explicit, informing participants that a sham intervention is involved.

The need to explicitly inform study participants can be especially problematic for acupuncture research. Acupuncture needling, as well as being difficult to mimic visually, also elicits distinct physical sensations. Control interventions such as inactive TENS or a placebo medication are likely to produce a different psychological response compared with acupuncture (19). In addition, sham procedures such as the recently devised noninvasive retractable 'stage dagger' needle may make it increasing difficult to deceive experienced acupuncture participants over a period of time (20). If participants are alerted to the possibility of receiving a sham or a placebo treatment at the informed consent stage, it may make it difficult to reliably blind the participants to the control intervention. This raises the question of whether partially informed consent can be ethical. Miller and Kaptchuk (21) argue strongly that 'accurate disclosure, consistent with informed consent, should take precedent over deceptive disclosure for the sake of maximizing scientific validity, especially in the absence of rigorous data showing that accurate disclosure undermines satisfactory blinding of participants'.

A recent study evaluated the ethical concerns over their use of partial consent in a randomized non-pharmacological study that used a placebo intervention (22). They reported that on completion of the study after the subjects had been informed of the intervention status, both groups agreed with the informed consent procedure. They further argue that partial consent may be justified if:

- the target illness does not demand urgent medical attention;

- the treatment is low risk;

- the risks have been adequately assessed and can be satisfactorily managed;

- the merit of the research far outweighs the risk to the participant;

- there is no known treatment for the target illness;

- the internal validity of the study is threatened (failure to blind);

- that participants are informed explicitly (in the consent form) that they would not receive any information on the specific goals of the interventions;

- participants are told that the specifics of the interventions will be given at the completion of the study.

The case for participant deception and partial consent needs further discussion and may be possible under specific conditions.

Another ethical concern often voiced is that randomized controlled trials (RCTs) ignore participant preference. This is particularly the case where CM has widespread accepted use. For example in China or Korea, acupuncture and herbal medicine are accepted as valid therapies and participants are reluctant to enter a randomized trial for fear of not receiving their treatment choice (23). A number of modified RCT designs that account for participant preferences have been suggested and tried in the West (24). These include the Baskerville design (Fig. 1) and the comprehensive cohort design (Fig. 2). The Baskerville design allows participants to choose whether they remain with the group they have been randomized to, cross over into the other group or withdraw from the trial. The comprehensive cohort design allows participants to chose, prior to randomization, whether they wish to be randomized. Those that choose not to be randomized are free to further decide whether they enter the treatment or control group. Those that agree to randomization are then randomized to either the treatment or the control group. These two designs, however, could be used only when participants are to be informed of their group allocation (e.g. wait list, active control). They could not be used when a sham or placebo control is used. In addition, it may be difficult to recruit sufficient numbers for the control group, with the distinct possibility that most participants would opt for the treatment group. A second possible problem is the unknown potential of allocation bias as a confounder when interpreting results from such trials. Interestingly, a recent review of 32 patient preference studies found little evidence that trials that use such designs 


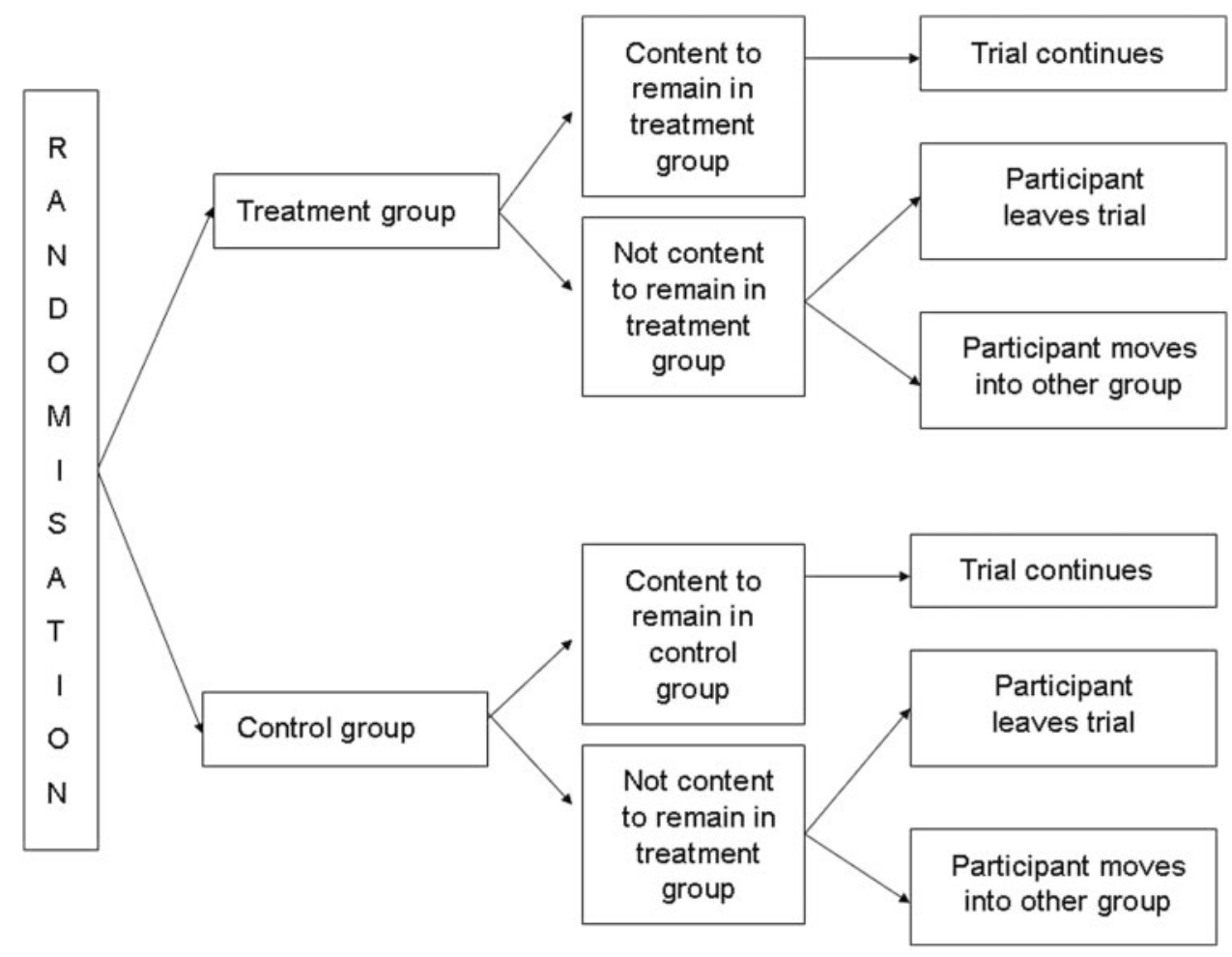

Figure 1. The Baskerville modified RCT design [adapted from Ernst (24)].

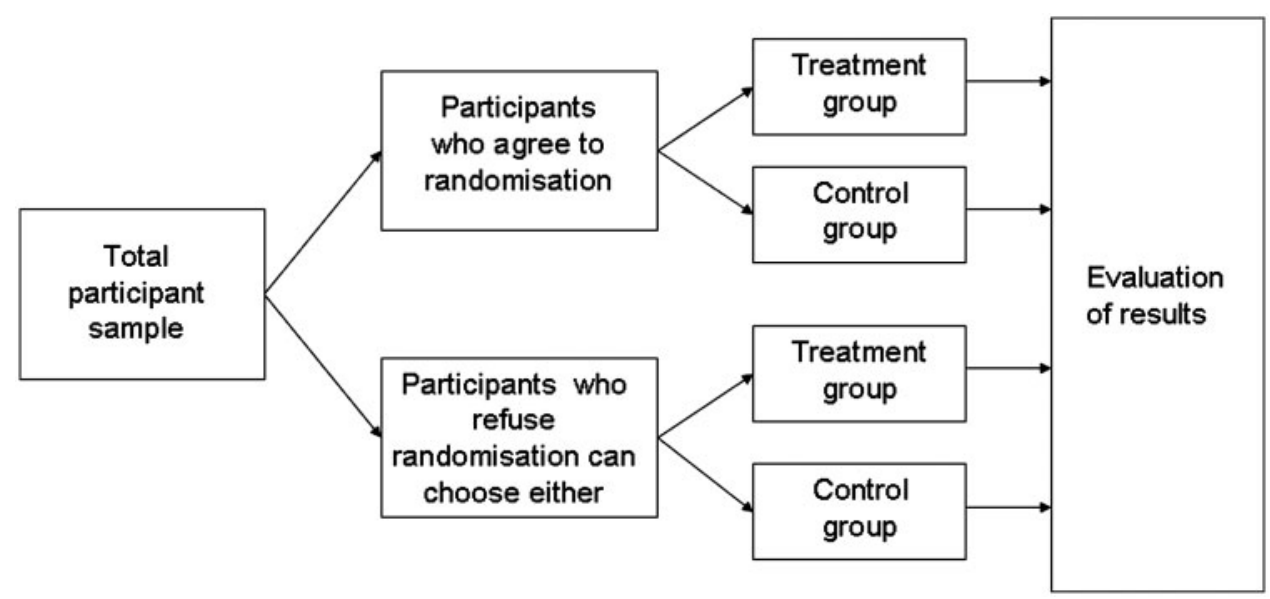

Figure 2. The comprehensive cohort design [adapted from Ernst (24)].

substantially interfere with the internal validity of randomized trials (25). Further research needs to be undertaken before widescale use of patient preference trials are undertaken to ensure that they do not introduce bias.

\section{Cross-cultural Research}

As globalization increases, the potential for international research collaboration will intensify. The acupuncture breech birth study conducted by Italian researchers using Chinese resources is a recent example (26). China, with its rich resources, human capital and its complex cultural interplay is a mixed blessing. China's potential is offset by her vulnerability with 'the rush to claim patents for and intellectual property rights to the resulting products and benefits fuels competition rather than scientific detachment and proper attention to the needs and claims of vulnerable groups' (27). To make it even more complex, the ongoing situation involves evaluating a process rather than its results. Chinese and Asian bioethicists continue to struggle to define, reframe and develop bioethics that is in harmony with China's cultural and moral inheritance. Drawing on Confucian, Buddhist and Taoist perspectives, many Chinese bioethicists argue that China's contemporary situation cannot be 
resolved by engaging Western bioethics that have resulted from very different cultural forces (28-30). Furthermore, the transition from a planned economy to a market economy will lead to further ethical reconsideration (31). With this perspective in mind, a number of problems arise when applying Western research ethics in an Asian culture.

One such difficulty concerns the cultural differences associated with obtaining informed consent (32). From the Western ethical perspective, the individual is seen as an autonomous agent with the ability to freely choose without consideration of others. Confucian ethics, on the other hand, perceive the individual as being situated within a complex familial (filial piety) and societal network, where the decision to become involved in a research study may often be influenced by relationships and responsibilities (33). It may be necessary to obtain consent from family members as well as the participant. This may especially be the case where the participant is a sick patient. Occasionally, the participant may not want to consent and family members agree or the patient may give consent but the family will not. Disputes may arise when the opinion of family members are neglected. Researchers may need to obtain a mutual agreement between participants and family members.

Furthermore, written consent may be difficult to obtain and it may be culturally insensitive to insist the participant sign a consent form. In Chinese culture, the signing of a consent form would be seen similar to signing oneself into indenture and many participants would rather orally consent than sign their name on paper (34). In this case, verbal consent can be obtained and formally documented or recorded.

If the consent cannot be obtained in writing, the non-written consent must be formally documented and witnessed.

The Helsinki Declaration (paragraph 22) (4)

Another potential issue is the use of placebo-controlled studies. Historically, the value system of Chinese medical ethics can be traced back to the Confucian precept that medicine is a humane art. Medicine is not only a means to help people during illness but a moral obligation to love people and relieve their suffering through personal caring and medical treatment (35). Use of a placebo is seen as depriving or cheating a patient of a valuable treatment and this has been reflected in the large number of uncontrolled cohort studies and single case reports in the Chinese medical literature and translated publications in the West (23). In the current evidence-based climate, such research designs are perceived to have little scientific merit and more appropriate designs such as outcome research may be more suitable.

Further exploration of cross-cultural ethics is required. Researchers need to be aware that the ethical values by which research is designed and conducted continue to respect the variety of opinion associated with different cultural perspectives.

\section{Weighting of Risk-Benefit}

The declaration of risks and benefits is an integral aspect of informed consent. Participants should be aware of the range and incidence of adverse events associated with their involvement in a research study. Again, the Declaration of Helsinki is explicit in this regard

Every medical research project involving human subjects should be preceded by careful assessment of predictable risks and burdens in comparison with foreseeable benefits to the subject or to others.

The Helsinki Declaration (paragraph 16) (4)

A number of recent reports have shown acupuncture to have a very low rate of adverse events associated with its practice. White (36), in reviewing the literature, reported on the outcomes of more than a million acupuncture treatments. He found that the risk of a serious adverse event was estimated to be $0.05 / 10000$ treatments and $0.55 / 10000$ individual patients. Similarly published reports have identified a number of side effects attributed to Chinese herbal medicine including stomach upset, skin rash and bleeding gums. Whilst Chinese herbs have been in common usage for at least 2000 years, the safety and the incidence of adverse events and their frequency is not well established (37). It is common for an ethics committee to require that herbal researchers report the known toxicological, pharmacological and adverse event data within their application in order to evaluate the risk-benefit ratio. While many of these reactions may be of minor concern, they should nevertheless be mentioned within the consent form (38).

In addition to informing participants of the risks, adverse events should also be monitored during the study. Participants should be asked at the completion of each intervention session, and prior to the following session, whether they experienced any undue reactions or uncomfortable sensations. All suspected adverse events should be reported and evaluated according to the likelihood that the acupuncture or herbal medicine contributed in eliciting them.

\section{Placebo Controls-An Ethical Alternative?}

One of the most controversial issues in research involves selection of the control intervention $(39,40)$. While the research question may be important in deciding what type of control condition is used, there are ethical concerns if a wait list or placebo control is employed. Both of these control conditions involve the delay or 
denial of standard medical care. In this day and age, very few diseases or illnesses do not receive at least partial benefit from standard medical care; therefore, the use of a placebo or a no-treatment control could be considered harmful and hence unethical. The 2000 Declaration of Helsinki is clear in enunciating this perspective (paragraph 29) (4):

The benefits, risks, burdens and effectiveness of a new method should be tested against those of the best current prophylactic, diagnostic, and therapeutic methods. This does not exclude the use of placebo, or no treatment, in studies where no proven prophylactic, diagnostic or therapeutic method exists.

Following the publication of the fifth revision of the Helsinki Declaration in 2000 (4), debate intensified concerning the use of placebo control groups especially when effective treatments are available. Opponents of the use of a placebo in a clinical trial argued that:

'every patient-including those of a control group, if any- should be assured of the best proven diagnostic and therapeutic method.' This statement effectively proscribes the use of a placebo as control when a 'proven' therapeutic method exists. The Helsinki declaration also directs that a study that violates its precepts should not be accepted for publication ...there is no straightforward way to estimate how many trials are undertaken that involve the unethical use of placebos.

Rothman and Michels (41)

The ensuing debate resulted in the World Medical Association in 2002 (4) publishing a note of clarification on paragraph 29 .

The WMA hereby reaffirms its position that extreme care must be taken in making use of a placebo-controlled trial and that in general this methodology should only be used in the absence of existing proven therapy. However, a placebocontrolled trial may be ethically acceptable, even if proven therapy is available, under the following circumstances:

- Where for compelling and scientifically sound methodological reasons its use is necessary to determine the efficacy or safety of a prophylactic, diagnostic or therapeutic method; or

- Where a prophylactic, diagnostic or therapeutic method is being investigated for a minor condition and the patients who receive placebo will not be subject to any additional risk of serious or irreversible harm.
Researchers are obliged to consider if the risks and burdens to the participant, from delaying or denying a proven therapeutic treatment, outweigh the research objectives. The Declaration of Helsinki is again quite clear in expressing this view (paragraphs 16 and 17) [4].

Every medical research project involving human subjects should be preceded by careful assessment of predictable risks and burdens in comparison with foreseeable benefits to the subject or to others...

Physicians should abstain from engaging in research projects involving human subjects unless they are confident that the risks involved have been adequately assessed and can be satisfactorily managed. Physicians should cease any investigation if the risks are found to outweigh the potential benefits

If the disease to be treated in the trial were chronic and stable, and the withholding of a proven therapeutic treatment constitutes a low risk to the participant, then the ethical concerns of using a placebo would be minimal. One strategy to ensure the participants were not denied effective treatment, especially if the acupuncture or herbal medicine was shown to be beneficial, is to offer the participant treatment at the completion of the trial. This strategy could be explicitly stated in the study protocol. The 2004 note of clarification to paragraph 30 (4) states that:

The WMA hereby reaffirms its position that it is necessary during the study planning process to identify post-trial access by study participants to prophylactic, diagnostic and therapeutic procedures identified as beneficial in the study or access to other appropriate care. Post trial access arrangements or other care must be described in the study protocol so the ethical review committee may consider such arrangements during its review.

If on the other hand, the disease was progressing quickly or had serious consequences if the proven therapeutic treatment was withheld, then the only ethical option would be to ensure the use of the proven therapeutic treatment in all arms of the trial. In this case, the acupuncture or herbal medicine treatment would be an adjunct to the standard treatment. The consideration of beneficence and non-maleficence remain central to the issue and the delay or withholding of a proven therapeutic treatment has to be balanced against the impact upon the participant's disease or state of health.

In summary, the consideration of ethics in the design and conduct of acupuncture and Chinese herbal medicine clinical trials is fundamental to good research. Ethical consideration of research involves the protection of the welfare and rights of the participants in the research. 
It also entails the development of rigorous research that will be of benefit to humankind. It is hoped that this paper will generate more questions on the role of ethics in $\mathrm{CM}$ clinical trial design.

\section{References}

1. Birch S. Clinical research on acupuncture: part 2. Controlled clinical trials, an overview of their methods. J Alternat Complement Med 2004;10:481-98.

2. Miller FG, Emanuel EJ, Rosenstein DL, Strauss SE. Ethical issues concerning research in complementary and alternative medicine. J Am Med Assoc 2004;291:599-604.

3. World Health Organization Regional Office for the Western Pacific. Guidelines for Clinical Research on Acupuncture. World Health Organization, Manila: Philippines, 1995.

4. World Medical Association Declaration of Helsinki. Available at www.wma.net/e/policy/b3.htm. Accessed 22 March 2008.

5. Schnyer RN, Wayne PM, Kaptchuk TJ, Cheng XM, Zhang ZZ, Stason WB. Standardization of individualized treatments in a randomized controlled trial of acupuncture for stroke rehabilitation. $J$ Altern Complement Med 2006;12:106-9.

6. Zaslawski CJ, Davis S. The ethics of complementary and alternative medicine research: a case study of traditional Chinese medicine at the University of Technology, Sydney. Monash Bioethics Rev 2005;24:52-61.

7. Freedman B. Equipoise and the ethics of clinical research. New Engl J Med 1987;317:141-5.

8. Fønnebø V, Grimsgaar S, Walach H, Ritenbaugh C, Norheim AJ, MacPherson $\mathrm{H}$, et al. Researching complementary and alternative treatments - the gatekeepers are not at home. BMC Med Res Methodol 2007;7:7.

9. Berman B, Lao L-X, Greene M, Anderson RW, Wong RH, Langenberg P, et al. Efficacy of traditional Chinese acupuncture in the treatment of symptomatic knee osteoarthritis: a pilot study. Osteoarthritis Cartilage 1995;3:139-42.

10. Berman BM, Singh BB, Langenberg P, Li H, Hadhazy V, Bareta J, et al. A randomised trial of acupuncture as an adjunctive therapy in osteoarthritis of the knee. Rheumatology 1999;38:346-54.

11. Berman BM, Lao L, Langenberg P, Lee WL, Gilpin AMK, Hochberg MC. Effectiveness of acupuncture as adjunctive therapy in osteoarthritis of the knee. Ann Intern Med 2004; 141:901-10.

12. Moher D, Schulz KF, Altman D. The CONSORT Statement: revised recommendations for improving the quality of reports of parallel-group randomized trials 2001. Explore (NY) 2005;1:40-5.

13. MacPherson H, White A, Cummings M, Jobst KA, Niemtzow RC. Standards for reporting interventions in controlled trials of acupuncture: the STRICTA recommendations. $J$ Altern Complement Med 2002;8:85-9.

14. Gagnier JJ, Boon H, Rochon P, Moher D, Barnes J, Bombardier C. Reporting randomized, controlled trials of herbal interventions: an elaborated CONSORT statement. Ann Intern Med 2006;144:364-7.

15. Bian ZX, Moher D, Dagenais S, Li YP, Wu TX, Liu L, et al. Improving the quality of randomized controlled trials in Chinese herbal medicine, part IV: applying a revised CONSORT checklist to measure reporting quality. J Chinese Integr Med 2006;4:233-42.

16. Journal of Negative Results in Biomedicine. Available at http:// www.jnrbm.com. Accessed 22 March 2008.

17. Ernst E. Research ethics in CAM. Focus Altern Complement Ther 2005;10:171-6.

18. Linde K, Dincer F. How informed is consent in shamcontrolled trials of acupuncture? J Altern Complement Med 2004;10:379-85.
19. Kaptchuk TJ, Stason WB, Davis RB, Legedza ATR, Schneyer RN, Kerr CE, et al. Sham device v inert pill: a randomised controlled trial of two placebo treatments. Br Med J 2006;332:391-7.

20. Tsukayama H, Yamashita H, Kimura T, Otsuki K. Factors which Influence the Applicability of Sham Needle in Acupuncture Trials: Two Randomised, Single Blind, Cross-Over Trials with Acupuncture-Experienced Subjects. 6th World Federation of Acupuncture-Moxibustion Societies Conference, Gold Coast, Australia, 2004.

21. Miller FG, Kaptchuk TJ. Acupuncture trials and informed consent. $J$ Med Ethics 2007;33:43-4.

22. Spigt MG, Knipschild PG, van Schayck CP, Knottnerus JA. The validity and ethics of giving placebo in a randomised non-pharmacological trial was evaluated. $J$ Clin Epidemiol 2005;58:350-6.

23. Xie Z. Clinical trials in traditional Chinese medicine. J Clin Ethics 2004;15:51-4.

24. Ernst E. RCTs for CAM. Focus Altern Complement Ther 2005;10:9-12.

25. King M, Nazareth I, Lampe F, Bower P, Chandler M, Morou M, et al. Impact of participant and physician intervention preferences on randomized trials. J Am Med Assoc 2005;293:1089-99.

26. Cardini F, Weixin H. Moxibustion for correction of breech presentation: a randomized controlled trial. $\mathrm{J}$ Am Med Assoc 1998;280:1580-4.

27. Doring O. Chinese researchers promote biomedical regulations: what are the motives of the biopolitical dawn in China and where are they heading? Kennedy Inst Ethics $J$ 2004;14:39-46.

28. Li Y-Z. Epistemological and methodological problems in studies of traditional Chinese ethical scholarship. New Literary Hist 1995;26:519-36.

29. Fan R. Reconstructionist Confucianism and health care: an Asian moral account of health care resource allocation. J Med Philos 2002;27:673-82.

30. Du Z-Z. On the development of teachers of medical ethics in China. The Hastings Centre Report 2000;30:S37-40.

31. Fan R-P. Towards a Confucian virtue ethics bioethics: reframing Chinese medical ethics in a market economy. Theor Med Bioethics 2006;27:541-66.

32. Dawson L, Kass NE. Views of US researchers about informed consent in international collaborative research. Social Sci Med 2005;61:1211-22.

33. Li B-F. Informed consent in research involving human subjects. $J$ Clin Ethics 2004;15:35-7.

34. Yuan L. Clinical trials in China: protection of subjects' rights and interests. J Clin Ethics 2004;15:30-4.

35. Zhang D-Q, Cheng Z-F. Medicine is a humane art. The basic principles of professional ethics in Chinese medicine. The Hastings Centre Report 2000;30:S8-12.

36. White A. A cumulative review of the range and incidence of significant adverse events associated with acupuncture. Acupuncture Med 2004;22:122-33.

37. Barnes J. Quality, efficacy and safety of complementary medicines: fashions, facts and the future. Part II: efficacy and safety. Br J Clin Pharmacol 2003;55:331-40.

38. Ioannnidis JPA, Evans SJW, Gotzsche PC, et al. Better reporting of harms in randomized trials: an extension of the CONSORT statement. Ann Intern Med 2004;141:781-8.

39. Vickers AJ, de Craen AJM. Why use placebos in clinical trials? A narrative review of the methodological literature. J Clin Epidemiol 2000;53:157-61.

40. Cleophas TJM. Clinical trials: specific problems associated with the use of a placebo-control group. J Mol Med 1995;73:421-4.

41. Rothman KJ, Michels KB. The continuing unethical use of placebo controls. New Engl J Med 1994;331:394-8.

Received October 25, 2007; accepted May 27, 2008 


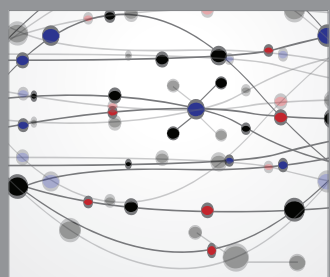

The Scientific World Journal
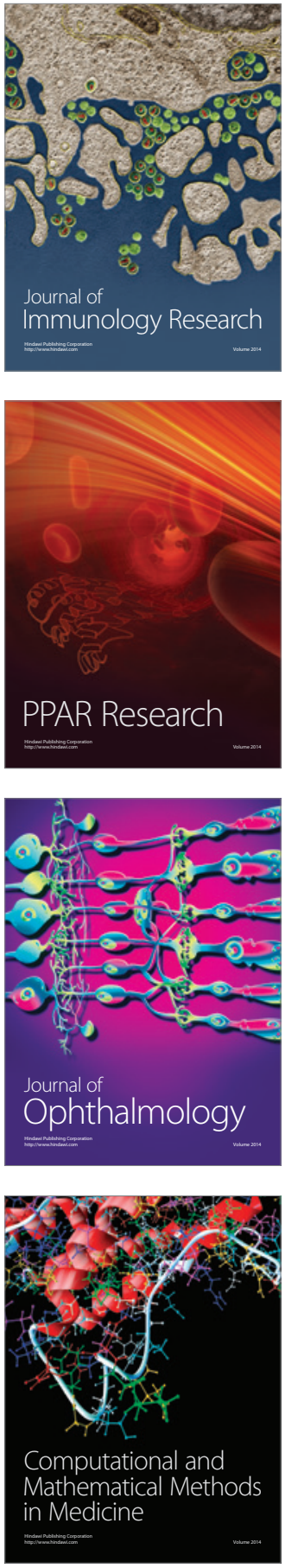

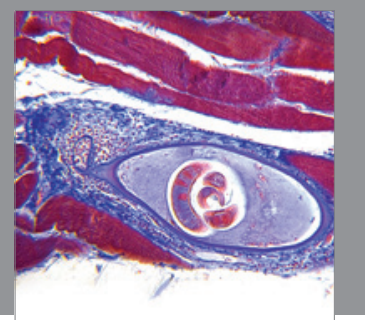

Gastroenterology

Research and Practice
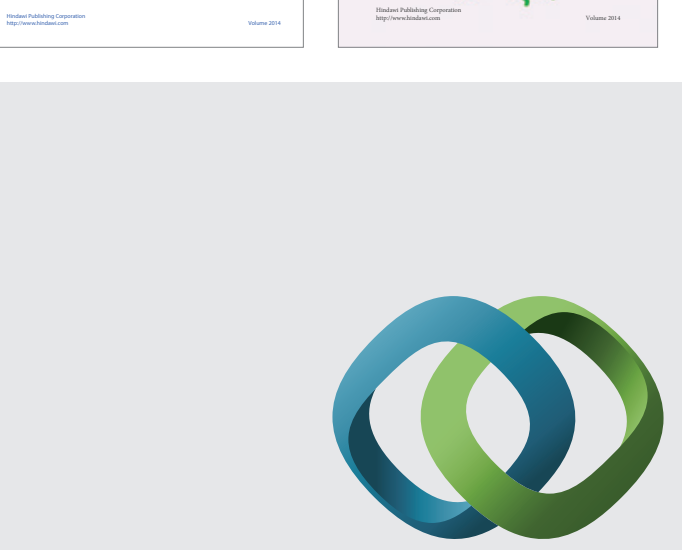

\section{Hindawi}

Submit your manuscripts at

http://www.hindawi.com
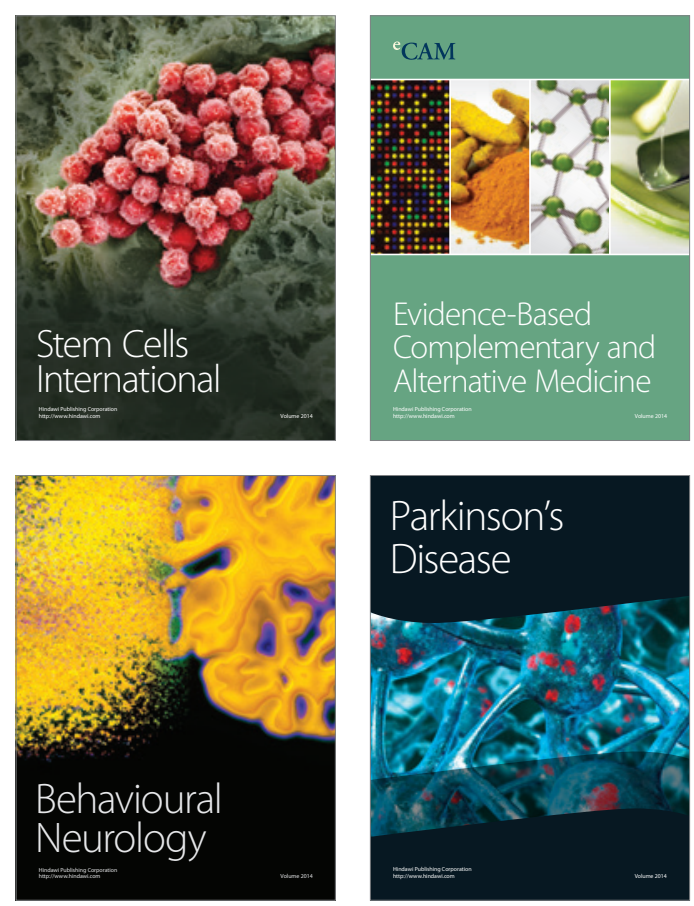

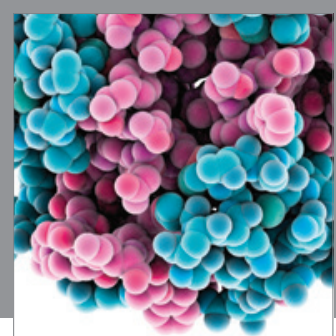

Journal of
Diabetes Research

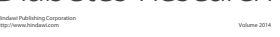

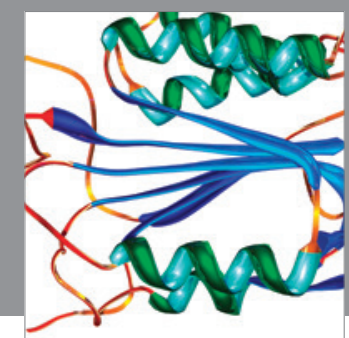

Disease Markers
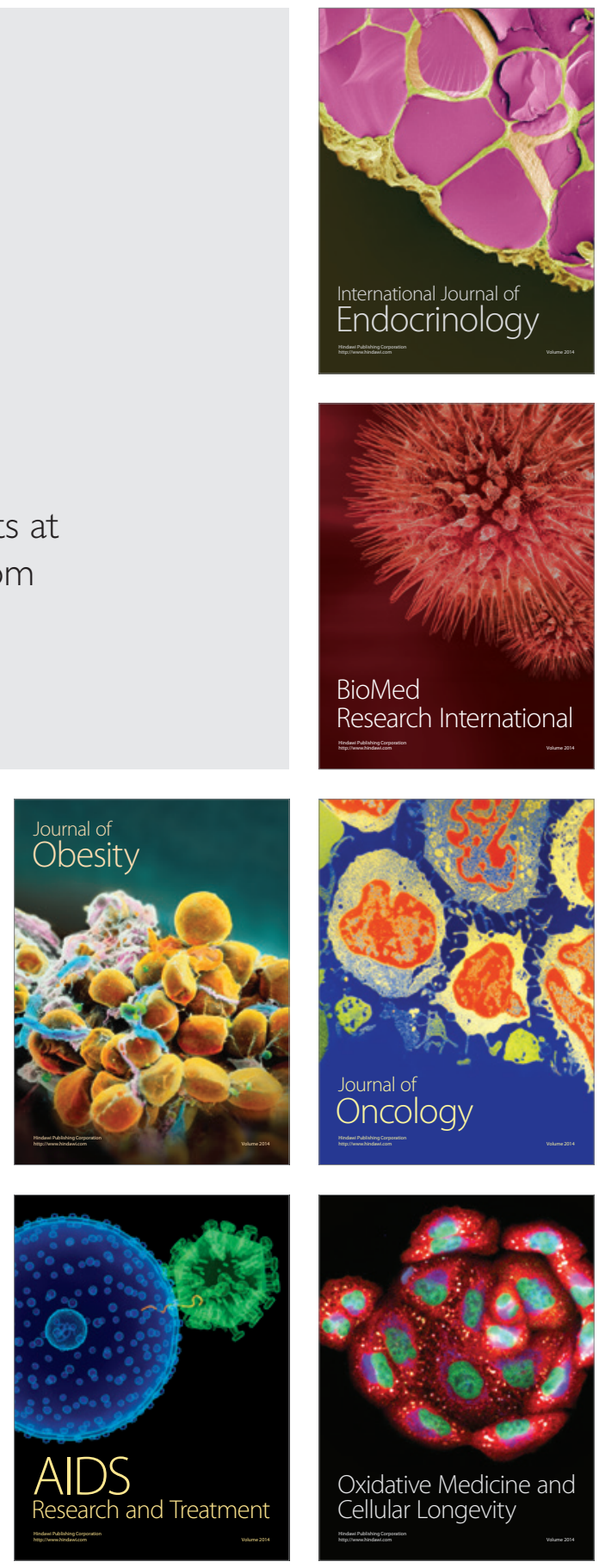